\title{
Genetic pre-screening for glaucoma in population-based epidemiology: protocol for a double-blind prospective screening study within Lifelines (EyeLife)
}

\author{
Anna Neustaeter ${ }^{1,2,3} \mathbb{B}$, Ilja Nolte ${ }^{2}$, Harold Snieder ${ }^{2,3}$ and Nomdo M. Jansonius ${ }^{1,3^{*}}$
}

\begin{abstract}
Background: Early detection of glaucoma is paramount to maintain patients' eyesight, however glaucomatous vision loss tends to begin in the periphery with up to $50 \%$ of patients unaware they are affected. Because glaucomatous vision loss is permanent, screening appears attractive, but currently is not cost-effective. Therefore we aim to investigate the utility of genetic pre-screening for glaucoma in a population-based setting, called EyeLife.

Methods: EyeLife adopts a double blind prospective design with contrasting groups. Selected participants ( $n=$ 1600) from the Lifelines cohort are 55 years of age or older, and of either the highest or lowest $20 \%$ of the genetic risk distribution for glaucoma. We obtained a highly curated list of genetic variants from the literature to obtain each participants' genetic risk for glaucoma. Participants will undergo comprehensive ophthalmic screening. The primary outcome is the relative risk of glaucoma given a high genetic risk compared to a low genetic risk.
\end{abstract}

Discussion: If genetic pre-screening is successful, it will increase the yield of a glaucoma screening program by focusing on high-risk individuals. This, in turn, may improve long-term visual health of middle-aged and elderly people.

Trial registration: Ethics approval was obtained on January 31, 2019, and the study was retrospectively registered with the Netherlands Trial Register (NL8718) on the 17th of June, 2020.

Keywords: Genetic risk score, Glaucoma, Screening, Prospective design, Lifelines

\section{Background}

Age-related ocular disorders like glaucoma contribute greatly to the burden of healthcare and loss of quality of life in older populations [1,2]. Glaucoma, a group of neurodegenerative diseases of the optic nerve, is the second-leading cause of irreversible vision loss in the world. Up to one half of those with glaucoma are

\footnotetext{
* Correspondence: n.m.jansonius@umcg.nl

1 Department of Ophthalmology, University of Groningen, University Medical Center Groningen, P.O.Box 30.001, 9700 RB Groningen, Netherlands

${ }^{3}$ Graduate School of Medical Sciences (Research School of Behavioural and

Cognitive Neurosciences), University of Groningen, Groningen, The Netherlands

Full list of author information is available at the end of the article
}

unaware of their disease status [3]. Adult-onset primary open-angle glaucoma is the most common subtype in Caucasians and within this article "glaucoma" refers to this subtype. Initial glaucomatous visual field loss (GVFL) may not be perceived as the brain perceptually masks visual field defects with visual features of nearby regions $[4,5]$. The slow course of the disease in combination with visual field defects initially in non-overlapping locations of each eye contribute to patient unawareness as well. Lowering the intraocular pressure [6-8](IOP), currently the only proven treatment modality, reduces end-of-life blindness from approximately 50 to $15 \%[6-8]$.

C C The Author(s). 2021 Open Access This article is licensed under a Creative Commons Attribution 4.0 International License, which permits use, sharing, adaptation, distribution and reproduction in any medium or format, as long as you give appropriate credit to the original author(s) and the source, provide a link to the Creative Commons licence, and indicate if changes were made. The images or other third party material in this article are included in the article's Creative Commons licence, unless indicated otherwise in a credit line to the material. If material is not included in the article's Creative Commons licence and your intended use is not permitted by statutory regulation or exceeds the permitted use, you will need to obtain permission directly from the copyright holder. To view a copy of this licence, visit http://creativecommons.org/licenses/by/4.0/. The Creative Commons Public Domain Dedication waiver (http://creativecommons.org/publicdomain/zero/1.0/) applies to the data made available in this article, unless otherwise stated in a credit line to the data. 
The combination of unawareness and the benefits of timely treatment suggest a pivotal role for screening.

Historical glaucoma screening programs were based exclusively on IOP measurements. However, only 10\% of untreated ocular hypertensive patients develop glaucoma within 5 years [9]. Additionally, in populations screened for glaucoma, only a minority of newly diagnosed patients had IOP outside the normal range at the time of screening [10-12]. Diurnal IOP fluctuation may partially explain this, but glaucoma still develops in those with IOP consistently within normal limits, a condition termed 'normal tension glaucoma'. Because IOP lowering is also effective in normal tension glaucoma, an increased vulnerability of the optic nerve head $(\mathrm{ONH})$ was hypothesized, with several reported factors contributing to increased vulnerability [13]. Other screening methods for glaucoma include testing functional vision via visual field (VF) testing, or assessing structural damage to the optic nerve or retinal nerve fiber layer (RNFL), typically with optical coherence tomography (OCT). However, despite technological advancements and detailed analyses, the poor screening performance of these individual techniques still limits applicability in the general population, due to the low prevalence of glaucoma $(\sim 2 \%$ in those aged 55 and older) [14]. With such a low prevalence, a single screening test requires a specificity of at least 95-97.5\% [15-17]. Using known glaucoma risk factors as a pre selection tool for screening aims to increase the prior probability of glaucoma in the population while limiting case-loss. People at an older age, of African descent, those with a family history of glaucoma, as well as those with myopia are at a higher risk of glaucoma [14, 18-20]. However, limiting glaucoma screening to only individuals with these risk factors is ineffective, as the majority of cases with one or more known glaucoma risk factors are detected in regular care [10, 21].

Accordingly, it is well-established that glaucoma and related endophenotypes are substantially heritable [22]. Inherited forms of glaucoma may be attributed to single gene mutations, like myocilin $(M Y O C)$ and optineurin $(O P T N)$, however these cases are rare and tend to occur early in life [23]. For the majority of glaucoma cases, genetic susceptibility arises from the combined effects of a number of genetic variants, each with individually small effects. These genetic variants can be combined into a single genetic risk score (GRS). Genome-wide association studies (GWAS) continually uncover novel genetic variants, termed single nucleotide polymorphisms (SNPs), associated with not only glaucoma risk and progression, but also continuous glaucoma endophenotypes like IOP, optic nerve properties [disc area (DA), cup area (CA), cupto-disc ratio (CDR)], central corneal thickness (CCT), retinal nerve fiber layer thickness (RNFLT), and peripapillary atrophy (PPA) [24-27]. A recent retrospective crosssectional cohort study reported that individuals at the top decile of genetic risk are at a 15 -fold increased risk in developing advanced glaucoma, compared to the bottom decile [26]. To date, the predictive ability of a glaucoma GRS used as a screening tool has not been validated [28] and the research question is: Are those at a higher genetic risk for glaucoma more likely to actually have glaucoma?

For this purpose, we aim to investigate the predictive ability of a glaucoma GRS. We extracted a highly curated list of SNPs that were genome-wide significantly associated with glaucoma and/or related-endophenotypes and combined them to create a glaucoma GRS. The GRS is employed in a population-based cohort as a pre-selection tool for glaucoma. We are currently conducting a doubleblind prospective study with contrasting genetic risk groups to evaluate the feasibility of genetic pre-screening for glaucoma case detection. The primary outcome is the relative risk of glaucoma for individuals at the highest versus the lowest $20 \%$ of the genetic risk distribution.

\section{Methods and analysis Study design}

This is a double-blind prospective study with contrasting genetic risk groups, investigating the predictive ability of a glaucoma GRS. It is based out of the ophthalmology department of the University Medical Center Groningen (UMCG), and called EyeLife. Participants are selected from Lifelines, a large prospective population-based cohort study of the Northern Netherlands [29]. It examines the health and health-related behaviours of 167,729 persons, in a unique three-generation design. Lifelines employs a broad range of investigative procedures in assessing the biomedical, socio-demographic, behavioural, physical, and psychological factors which contribute to the health and disease of the general population, with a special focus on multi-morbidity and complex genetics [30]. The cohort structure is described fully elsewhere [31]. Genetic information is currently available for more than 50,000 participants, and a subset of this population is invited to take part in EyeLife.

\section{Characteristics of participants}

Lifelines participants at either the highest or lowest 20\% of the glaucoma genetic risk distribution with signed informed consents are eligible to participate in EyeLife. In total, 1600 genotyped Lifelines participants 55 years of age and older, and amenable to additional research studies, are selected for participation in EyeLife.

\section{Genotype information}

Genotypic information for Lifelines was obtained in two phases. Initially 15,638 unrelated individuals within Lifelines were genotyped using the Illumina CytoSNP 12 v2 array (Illumina, San Diego, CA, USA). Initial quality control was carried out in PLINK [32]. Markers with a 
call rate $<95 \%$, Hardy-Weinberg equilibrium $p<0.0001$, or minor allele frequency $<1 \%$ were excluded. Samples with sex-mismatches, deviating heterzygosity ( $>4$ standard deviations from the mean), non-European ancestry, a call rate of $<95 \%$, duplicated samples, or first degree relatives were excluded. A total of 268,407 autosomal genetic markers, and 13,436 samples remained. Imputation was subsequently carried out using Beagle v3.1.0 [33] with the HapMap Phase 2 CEU reference panel (release 24, build 36) [34, 35].

Next, the UMCG Genetics Lifelines Initiative (UGLI) genotyped 38,030 additional Lifelines participants using the Infinium Global Screening Array ${ }^{\circ}$ (GSA) MultiEthnic Disease Version. Quality control focused on the autosomal and X chromosomes (691,072 markers). First, duplicate markers were removed, then samples and markers of low quality were filtered using call rate thresholding in two stages (80 and 99\%, respectively). Following that, variants that deviated significantly from Hardy-Weinberg equilibrium $\left(p<1 \times 10^{-6}\right)$ or that were monomorphic (minor allele frequency $=0$ ) were removed. Next, samples with a deviating heterozygosity ( $>4$ standard deviations from the expected mean adjusted for observed runs of homozygosity), unexpected duplicate samples, and samples of non-European ethnicity were excluded. After passing initial quality control, 36,339 samples and 571,420 genetic markers were imputed through the Sanger Imputation Service (http://imputation. sanger.ac.uk) utilizing the Haplotype Reference Consortium panel (http://www.haplotype-reference-consortium.org).

\section{Glaucoma genetic risk score}

Genome-wide association analyses indicate which SNPs are associated with an outcome of interest; outcomes can be binary such as glaucoma, or continuous like IOP. As there are millions of regressions per GWAS, a global threshold of $p \leq 5^{*} 10^{-8}$ was established for genome-wide significance [36]. A GRS is the genetic profile of SNPs that increase the risk of the disease or trait. It can be unweighted via counting the number of risk alleles, or weighted by the GWAS effect sizes of the SNPs included. The SNP effect direction is aligned so included SNPs represent an increased risk. The generalized GRS equation is:

$$
G R S=\Sigma_{i=1}^{N} \omega_{i} \cdot \chi_{i}
$$

where $N$ is the number of SNPs in the GRS, $\omega_{i}$ is the weight for SNP $i$ (in an unweighted GRS $\omega_{i}$ is 1 ; in a weighted GRS $\omega_{i}$ is the regression coefficient of SNP $i$ derived from GWAS summary statistics with glaucoma as the outcome - we used the effect size with glaucoma as the outcome, even where the SNP was originally genome-wide significant for an endophenotype), and $X_{i}$ is the allelic dosage of the risk variant $i$ [35]. We created and employed a weighted GRS as they explain more variance in complex disorders like glaucoma [35].

The glaucoma GRS used in this study is novel as it utilizes genome-wide significant SNPs from both glaucoma in addition to related endophenotypes to create a highly curated glaucoma GRS. We aim to improve upon current SNP-based glaucoma risk prediction by incorporating endophenotype-associated SNPs and applying the GRS prospectively in a population-based setting as a pre-screening tool. What follows below are the details of the approach.

A literature search was performed on November 8th, 2018 for GWAS of glaucoma and relevant endophenotypes (IOP, CCT, optic disc parameters, RNFL, and PPA) in populations of European ancestry, see Fig. 1 for a flow diagram of article and SNP selection. Initially, 134 articles were obtained. Articles were excluded $(n=93)$ for the following reasons: duplicates, the outcome was not primary/open-angle glaucoma, the population was not of European ancestry (mixed ethnicity GWAS were permitted if European ancestry was included), the GWAS was performed in children, the study design was gene-centric study designs, or it was a twin study. The full text of 42 articles was assessed, and articles regarding pseudoexfoliation glaucoma $(n=15)$ were further excluded. From these 27 articles, 732 variants were genome-wide significantly associated $\left(p \leq 5 \times 10^{-8}\right)$ with glaucoma or related endophenotypes. Duplicated SNPs $(n=142)$ were excluded, resulting in 590 SNPs. Next, the 590 SNPs were looked up in the summary statistics of Choquet et al. [27], a large glaucoma meta-GWAS combining the UK Biobank and GERA cohorts, to obtain SNP effect sizes. We always utilized the effect size from the glaucoma meta-GWAS, even where the SNP was originally genome-wide significant for an endophenotype. Of the 590 SNPs, 67 lacked summary statistic information, we obtained proxies using a linkage disequilibrium (LD) threshold of $r^{2} \geq 0.7$ for 41 SNPs within the Choquet summary statistics, totaling 564 SNPs [37]. In total, including the 47 lead SNPs from the Choquet meta-GWAS, we obtained 611 literature-derived variants containing summary statistic information [27]. Next, when a single study reported multiple significant dependent variants within a locus, we chose the most significantly reported variant. When multiple articles reported different and dependent variants in the same locus (LD threshold $r^{2} \geq 0.1$ ), the variant from the study with the largest sample size was selected (while preserving the lead SNPs), resulting in 268 independent SNPs used for the glaucoma GRS $[35,37]$.

To obtain the glaucoma GRS, the individual SNP effect sizes were multiplied by the allelic dosages and summed across all 268 SNPs (Eq. 1) for all participants. Individuals at the highest and lowest $20 \%$ of the genetic risk distribution are invited to participate in EyeLife. 


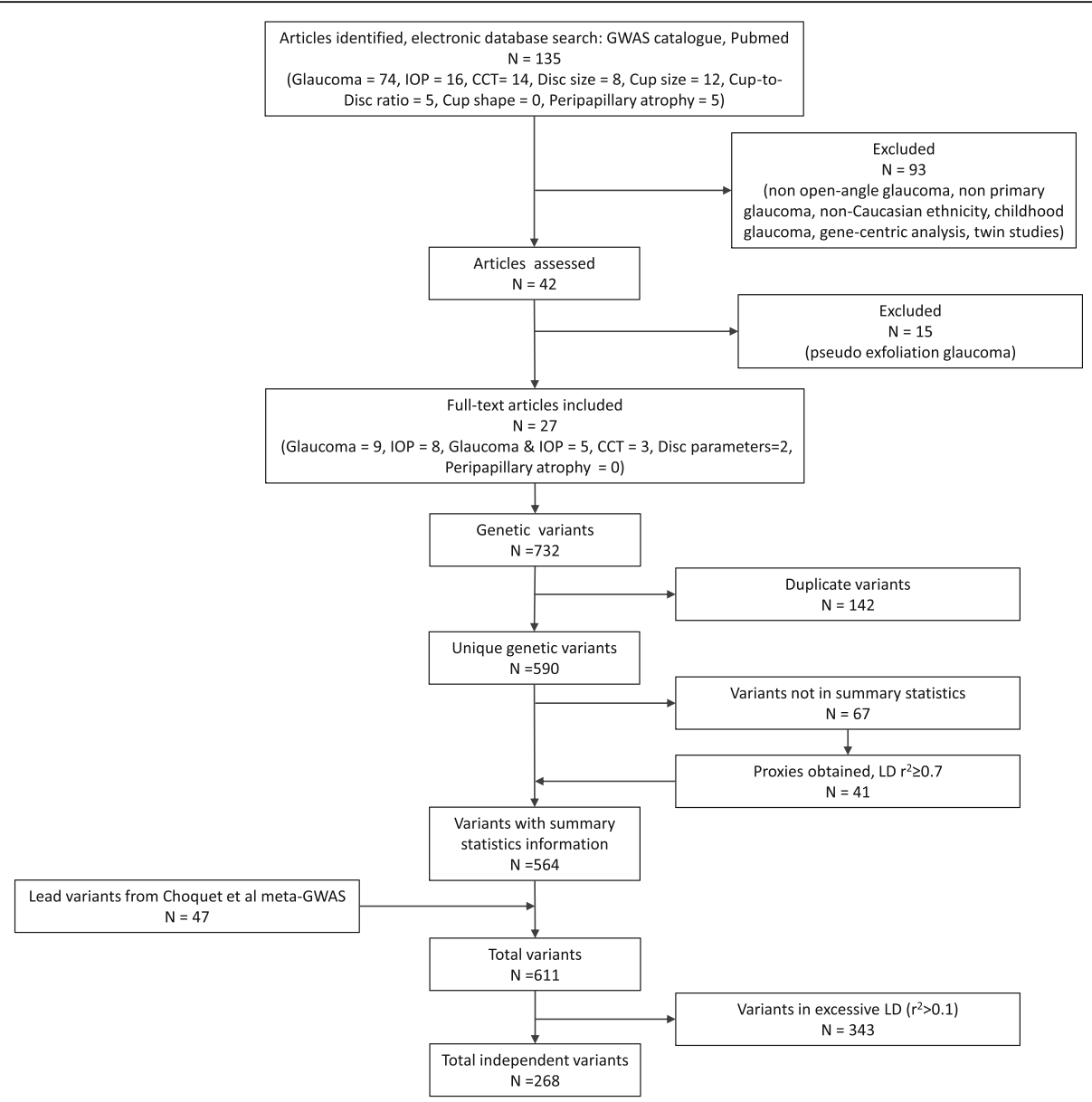

Fig. 1 Glaucoma genetic risk score flow diagram. The decision tree shows the article and genetic variant selection to obtain relevant SNPs for the glaucoma GRS

\section{Data gathering, interpretation, and phenotyping}

EyeLife participants undergo a series of ocular examinations. Figure 2 presents a flow diagram of participant selection, the EyeLife visit, and visit outcomes. Examinations were chosen for being non-contact, non-mydriatic, rapid, robust, easy-to-operate, and of proven value in glaucoma screening/research in population-based settings.

First, IOP is measured with the non-contact Ocular Response Analyzer (ORA; Reichert Ophthalmic Instruments, Inc., Buffalo, NY, USA) [38]. IOP is measured in both eyes; the measurement is repeated until the quality metric, wavefront score (WS), is above 6 , with a maximum of two measurements per eye. We record the cornealcompensated IOP (IOPcc), corneal hysteresis $(\mathrm{CH})$, and the Goldmann-correlated IOP (IOPg) of the measurement with the higher WS value.

Next, participants' visual acuity and refraction are obtained via the Nidek ARK-1S (NIDEK CO., LTD., Gamagori, Japan) [39]. Autorefraction is an average of three measurements, and is followed by visual acuity measured in three luminance conditions: high luminance $\left(200 \mathrm{~cd} / \mathrm{m}^{2}\right.$; best corrected visual acuity [BCVA]), low luminance acuity $\left(10 \mathrm{~cd} / \mathrm{m}^{2}\right)$, and acuity with a built-in glare source. The device also obtains pupil diameter and keratometry.

The visual field is tested using the Zeiss Humphrey frequency doubling technique (FDT) with the C-20-1 screening mode (Carl Zeiss Meditec, Jena, Germany) [40-44]. The test is repeated with any abnormal test location $P<0.01$. Visual field loss (VFL) is defined as the presence of one or more reproducibly abnormal test locations. Then GVFL is defined as the presence of VFL that is not explained by abnormalities in the fundus images, and excluding homonymous or bitemporal defects.

Following, the structural layers of the eye are taken via the Optopol OCT, NX700 (OPTOPOL technology, Zawiercie, Poland). We image the optic disc region, the macular area, and the anterior chamber including the cornea. For the optic disc region, we focus on the peripapillary RNFL (pRNFL); aberrations in the pRNFL are defined as clock-hour defects at $1 \%$ of age-, gender-, and ethnicity-based normative data in the inferior, superior, 


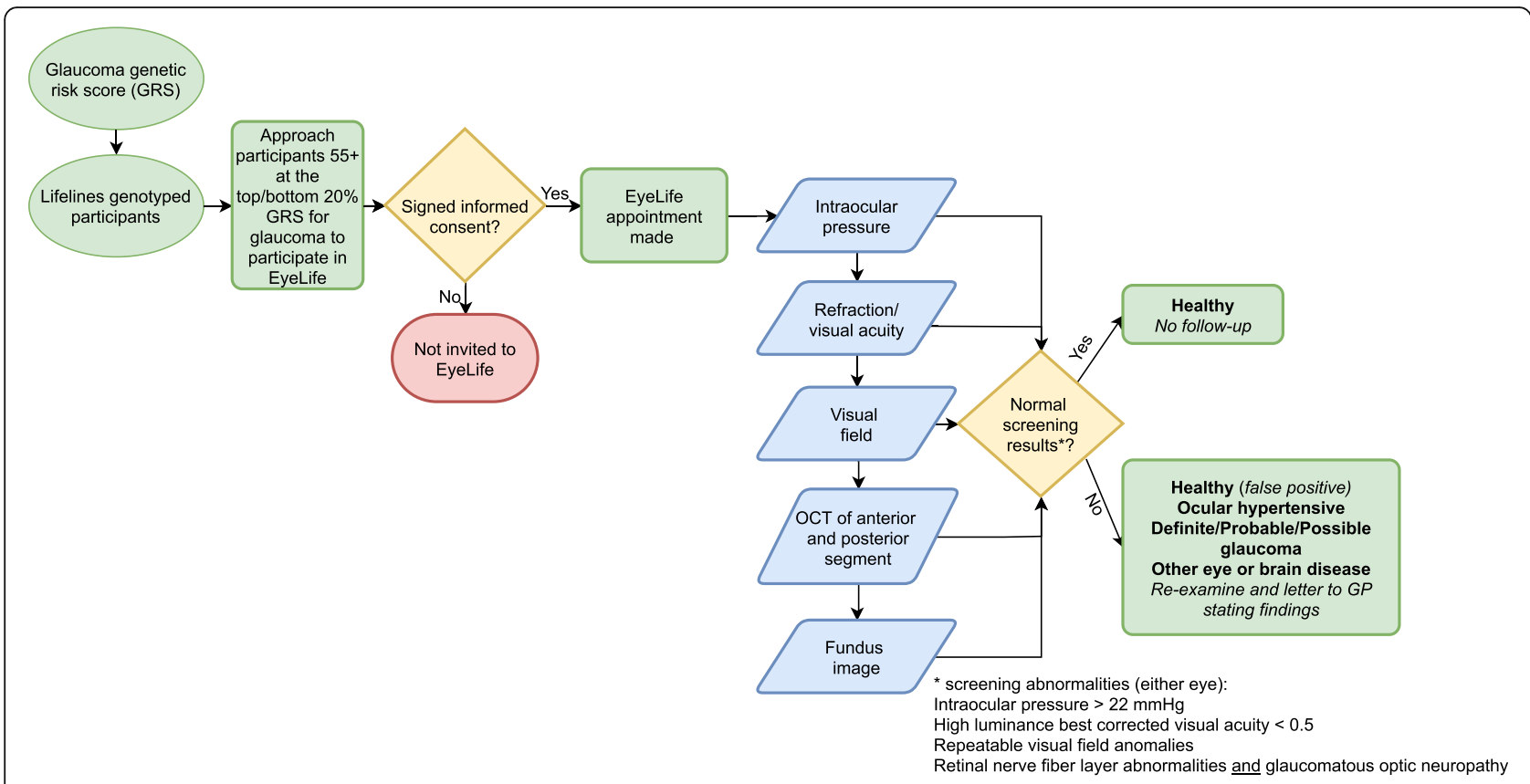

Fig. 2 Flow diagram of EyeLife. The selection process, ocular examinations, and decision tree for EyeLife findings is described

or temporal clock-hours [45]. Central corneal thickness (CCT) is measured via an anterior segment OCT scan and an averaged value of both eyes is obtained.

Finally, fundus photographs are obtained via the Nidek AFC-330 (NIDEK CO., LTD., Gamagori, Japan) where two 45-degree angle-of-view images are taken of both eyes, one centered on the optic disc and one centered on the macula. The optic disc is assessed from fundus images by a glaucoma specialist (NJ). Glaucomatous optic neuropathy (GON) is defined as either a vertical cup-disc ratio (VCDR) of at least 0.7 (the 97.5th percentile in the general population), [46] or notching (focal absence of the neural rim) inferiorly or superiorly, or the presence of a peripapillary splinter haemorrhage [46-48].

We invite participants for re-examination by an ophthalmologist with at least one of the following findings: (1) IOPcc $>22 \mathrm{mmHg}$ in either eye; (2) high luminance $\mathrm{BCVA}<0.5$ in either eye, unless there is a clear history of amblyopia or the participant is being treated for agerelated macular degeneration; (3) VFL in either eye; and (4) RNFL abnormalities together with GON.

\section{Glaucoma classification}

The International society of Geographical and Epidemiological Ophthalmology (ISGEO) classification is used as an initial reference point in defining glaucoma within EyeLife [48]. We classify EyeLife participants as definite, probable, or possible glaucoma, or healthy, similar to the Rotterdam Study [48]. We made two modifications to the ISGEO classification system. First, we removed the second GON cut-off point, the 99.5th percentile, for statistical reasons [49]. Second, currently RNFL assessment by OCT or other techniques is not within the ISGEO classification system, thus we modified classification according to the Northern Finland birth cohort eye study. They employed a 'two-of-three' (VF, RNFL, optic disc) method to classify glaucoma [50]. The resultant glaucoma categories are:

Definite glaucoma (combined functional and structural loss; the equivalent of ISGEO Category 1 diagnosis): GVFL with either RNFL abnormalities or GON;

Probable glaucoma (functional or confirmed structural loss): either GVFL (called 'field suspect' by the ISGEO) or RNFL abnormalities together with GON;

Possible glaucoma (structural loss): either RNFL abnormalities or GON (called 'disc suspect' by the ISGEO).

A glaucoma "case" is then defined as either definite or probable glaucoma. Missing data due to, for example, small pupils or media opacities, are interpreted as "normal findings" with other ocular tests examined in this context. For example, given the classifications above, a participant with GVFL, a normal fundus image, and missing OCT data is classified as probable glaucoma, based on the GVFL. Glaucoma is considered open-angle glaucoma (OAG) if an open-angle was found during gonioscopy at the re-invite (trabecular meshwork visible in at least 3 of 4 quadrants without compression); OAG was considered primary $O A G$ (POAG) if there were no signs of pigment dispersion or pseudoexfoliation, or (a history of) other eye diseases that may have caused glaucoma. Intraocular pressure is not taken into account in the glaucoma diagnosis, however an IOPcc $>22 \mathrm{mmHg}$ 
during the initial screening alongside an applanation tonometry (AT) reading above $20 \mathrm{mmHg}$ during the reinvite is defined as ocular hypertension (OHT), if no GVFL, RNFL abnormalities, or GON is present. Although CCT is not taken into account in the glaucoma or OHT diagnosis, it is considered in clinical follow-up. A clinical follow-up (via GP referral) is offered to those with definite glaucoma, or in cases of reproducibly elevated IOP (IOPcc $>22 \mathrm{mmHg}$ and $\mathrm{AT}>20 \mathrm{mmHg}$ ) with or without probable or possible glaucoma. We only refer OHT cases with CCT $\geq 600 \mu \mathrm{m}$ in combination with AT $>25 \mathrm{mmHg}$.

\section{Sample size}

Assuming an odds ratio of 3.5, based on a published odds ratio for family history, [51] and a total population prevalence of glaucoma of 3.2\% [46] for our target group of participants aged 55 plus (the prevalence in the top $20 \%$ genetic risk distribution of $5.0 \%$ and a prevalence of $1.5 \%$ in the bottom $20 \%$ genetic risk distribution), a power of 0.80 , and an alpha of 0.008 we will be able to detect differences as small as 0.15 standard deviations for continuous traits with 660 subjects per risk group, which are clinically relevant effect sizes for glaucoma endophenotypes of interest; IOP, CCT, RNFL, and optic disc parameters (power calculator PASS11; Hintze, J. (2011). PASS 11. NCSS, LLC. Kaysville, Utah, USA. www.ncss.com.) [25]. With an assumed drop-out rate of $20 \%$, we will invite 800 participants per risk group. Given the above assumptions, we estimate approximately 33 glaucoma cases (where a case is either definite or probable glaucoma) will be found in the participants constituting the top $20 \%$ of the genetic risk distribution for glaucoma, and nine cases in those at the bottom $20 \%$ of the genetic risk distribution.

\section{Selection, blinding, and minimization of bias}

Using the glaucoma GRS, we identified 8279 Lifelines participants at the top, and 8199 participants at the bottom $20 \%$ of the genetic risk distribution for glaucoma. Subsequently, Lifelines refines this list based on age (55+ ), proximity to the research centre (postcodes 93009999), and participation status (if the participant is alive, still actively participating in Lifelines research, and amenable to additional studies). Lifelines sends this refined list of selected eligible participants to a third party to draw 800 participants at random from the top $20 \%$ of the genetic risk distribution and 800 from the bottom $20 \%$. Participants are anonymized with a unique EyeLife ID. During the visit only the EyeLife ID, age, gender, and ethnicity of the participant is recorded.

In order to minimize bias, researchers, data collectors and participants were blinded to the genetic profile of participants throughout data collection. Any associations between genetic profile and outcome of the glaucoma assessment will be analysed after data collection is completed.

\section{Data analysis}

The primary outcome is the relative risk of glaucoma (definite or probable) given a high genetic risk, see Table 1.

The glaucoma relative risk (RR) is then:

$$
R R=\frac{a /(a+b)}{c /(c+d)}
$$

Following, the standard error (SE) of the log to the base $e$ of the relative risk is then:

$$
\mathrm{SE}\{\ln (\mathrm{RR})\}=\sqrt{\frac{1}{a}+\frac{1}{c}-\frac{1}{a+b}-\frac{1}{c+d}},
$$

and the associated 95\% confidence interval (CI):

$$
\begin{aligned}
95 \% C I=\exp (\ln (R R)- & 1.96 * S E\{\ln (R R)\}) \text { to } \exp (\ln (R R) \\
+ & 1.96 * S E\{\ln (R R)\})
\end{aligned}
$$

The secondary outcomes are to assess if glaucoma endophenotypes (IOP, DA, CA, CDR, CCT, and RNFLT) are significantly different between high and low glaucoma genetic risk distribution.

\section{Discussion}

As the awareness of glaucoma status hovers at approximately $50 \%$, an effective population-based screening procedure for glaucoma will enable earlier diagnoses and treatment thus preserving ocular health. There is an increased interest in the explanatory power of genetic variants to disease risk, as those at the higher end of the genetic risk distribution could be more intensively monitored, i.e. personalized screening, affording early detection and prevention. A recent report investigated the power of glaucoma detection with GRS that include endophenotypes in a case-control cohort $[26,52]$. Although the explanatory power of a glaucoma genetic risk score is promising, thus far it is applied retrospectively. This study employs a glaucoma GRS

Table 1 Data outcome table of genetic risk and glaucoma of EyeLife

\begin{tabular}{llll}
\hline & High genetic risk & Low genetic risk & Total \\
\hline Glaucoma & $\mathrm{a}$ & $\mathrm{c}$ & $\mathrm{a}+\mathrm{c}$ \\
No glaucoma & $\mathrm{b}$ & $\mathrm{d}$ & $\mathrm{b}+\mathrm{d}$ \\
Total & $\mathrm{a}+\mathrm{b}$ & $\mathrm{c}+\mathrm{d}$ & $\mathrm{a}+\mathrm{b}+\mathrm{c}+\mathrm{d}$ \\
\hline
\end{tabular}


prospectively to investigate its promise and feasibility as a pre-screening tool.

If this method is found to be an effective prescreening approach for glaucoma in a population-based cohort, the next steps include optimizing the GRS with up-to-date SNPs implicated with glaucoma risk, including relevant endophenotypes, confirming our findings in a second population-based cohort, and extending the approach to different ethnic groups. The eventual goal is to detect glaucoma cases as early as possible to maintain eyesight for as long as possible.

\begin{abstract}
Abbreviations
AT: Applanation tonometry; BCVA: Best corrected visual acuity; CCT: Central corneal thickness; Cl: Confidence interval; IOPcc: Corneal compensation IOP; Ch: Corneal hysteresis; CA: Cup area; CDR: Cup-to-disc ratio; DA: Disc area; FDT: Frequency doubling technique; GRS: Genetic risk score; GWAS: Genomewide association study; GON: Glaucomatous optic neuropathy; GFVL: Glaucomatous visual field loss; GSA: Global Screening Array; IOPg: Goldmann-correlated IOP; ISGEO: International society of Geographical and Epidemiological Ophthalmology; IOP: Intraocular pressure; LD: Linkage disequilibrium; OHT: Ocular hypertension; ORA: Ocular Response Analyzer; OAG: Open angle glaucoma; ONH: Optic nerve head; OCT: Optical coherence tomography; PPA: Peripapillary atrophy; pRNFL: peripapillary RNFL; POAG: Primary open angle glaucoma; RR: Relative risk; RNFL: Retinal nerve fiber layer; SNP: Single nucleotide polymorphism; SE: Standard error; SOP: Standard operating procedure; UGLI: UMCG Genetics Lifelines Initiative; UMCG: University Medical center Groningen; VCDR: Vertical cup-disc ratio; VF: Visual field; VFL: Visual field loss; WS: Wavefront score
\end{abstract}

\section{Acknowledgements}

Not applicable.

\section{Roles and responsibilities}

The study will be managed by a research team based in the ophthalmology and genetic epidemiology departments in the UMCG. The research team is responsible for leading the development of standard operating procedures (SOPs) for data collection, training data collectors, and evaluating participants' glaucoma status.

\section{Study schedule and status}

Data collection commenced on the 29th of April, 2019 and is ongoing until adequate power is obtained for final analysis. Due to COVID-19, data collection was suspended on the 13th of March, 2020, and commenced again on the 8th of September, 2020.

\section{Authors' contributions}

The study concept and design was conceived by NMJ, HS, and AN. Data collection and screening will be performed by AN and NMJ. Genetic risk score was developed by AN, HS, and IN. Data analysis will be performed by AN, NMJ, and HS. AN drafted the manuscript with edits and critical revisions from NMJ, HS, and IN. All authors read and approved the final manuscript.

\section{Funding}

This project has received funding from the European Union's Horizon 2020 research and innovation programme under the Marie Skłodowska-Curie grant agreement No 661883 (EGRET) and from the Rotterdamse Stichting Blindenbelangen, grant number B20150036. The funding organization had no role in the design, conduct, analysis, or publication of this research. The Lifelines Biobank initiative has been made possible by subsidy from the Dutch Ministry of Health, Welfare and Sport, the Dutch Ministry of Economic Affairs, the University Medical Center Groningen (UMCG the Netherlands), University of Groningen and the Northern Provinces of the Netherlands.

\section{Availability of data and materials}

The data that support the findings of this study are available from Lifelines but restrictions apply to the availability of these data, which were used under license for the current study, and so are not publicly available. Data are however available from the authors upon reasonable request and with permission of Lifelines.

\section{Ethics approval and consent to participate}

Lifelines obtained ethical approval from the ethics board of the University Medical Center Groningen (UMCG) for population-based data collection on the 15th of October, 2007. For this study, the ethics board of the UMCG approved the study protocol on the 31st of January, 2019. This study was registered with the Netherlands Trial Register (NL8718). All participants provided written informed consent. The study follows the tenets of the Declaration of Helsinki and is being monitored by the UMCG. Results will be disseminated once data collection is complete.

\section{Consent for publication}

Not applicable.

\section{Competing interests}

The authors declare that they have no competing interests.

\section{Author details}

${ }^{1}$ Department of Ophthalmology, University of Groningen, University Medical Center Groningen, P.O.Box 30.001, 9700 RB Groningen, Netherlands. ${ }^{2}$ Department of Epidemiology, University of Groningen, University Medical Center Groningen, Groningen, The Netherlands. ${ }^{3}$ Graduate School of Medical Sciences (Research School of Behavioural and Cognitive Neurosciences), University of Groningen, Groningen, The Netherlands.

Received: 2 December 2020 Accepted: 15 December 2020

Published online: 07 January 2021

\section{References}

1. Rouland J-F, Berdeaux G, Lafuma A. The Economic Burden of Glaucoma and Ocular Hypertension. Drugs Aging. 2005;22:315-321. https://doi.org/10.2165/ 00002512-200522040-00004.

2. Quaranta L, Riva I, Gerardi C, Oddone F, Floriani I, Konstas AGP. Quality of life in Glaucoma: a review of the literature. Adv Ther. 2016;33(6):959-81.

3. Bourne RRA, Stevens GA, White RA, Smith JL, Flaxman SR, Price $H$, et al. Causes of vision loss worldwide, 1990-2010: a systematic analysis. Lancet Glob Health. 2013;1:e339-49. https://doi.org/10.1016/s2214-109x(13)70113-x.

4. Gupta P, Zhao D, Guallar E, Ko F, Boland MV, Friedman DS. Prevalence of Glaucoma in the United States: the 2005-2008 National Health and nutrition examination survey. Invest Ophthalmol Vis Sci. 2016;57(6):2905-13.

5. Komatsu H. The neural mechanisms of perceptual filling-in. Nat Rev Neurosci. 2006;7:220-31. https://doi.org/10.1038/nrn1869.

6. Peters $D$, Bengtsson B, Heijl A. Factors associated with lifetime risk of openangle glaucoma blindness. Acta Ophthalmol. 2014;92(5):421-5.

7. van Gestel A, Severens JL, Webers CAB, Beckers HJM, Jansonius NM, Schouten JSAG. Modeling complex treatment strategies: construction and validation of a discrete event simulation model for glaucoma. Value Health. 2010;13(4):358-67.

8. Ernest PJG, Busch MJWM, Webers CAB, Beckers HJM, Hendrikse F, Prins MH, et al. Prevalence of end-of-life visual impairment in patients followed for glaucoma. Acta Ophthalmol. 2013;91(8):738-43.

9. Kass MA, Heuer DK, Higginbotham EJ, Johnson CA, Keltner JL, Miller JP, et al. The ocular hypertension treatment study: a randomized trial determines that topical ocular hypotensive medication delays or prevents the onset of primary open-angle glaucoma. Arch Ophthalmol. 2002;120(6): 701-13 discussion 829-30.

10. Stoutenbeek R, de Voogd S, Wolfs RCW, Hofman A, de Jong PTVM, Jansonius NM. The additional yield of a periodic screening programme for open-angle glaucoma: a population-based comparison of incident glaucoma cases detected in regular ophthalmic care with cases detected during screening. Br J Ophthalmol. 2008;92(9):1222-6.

11. Dielemans I, Vingerling JR, Wolfs RCW, Hofman A, Grobbee DE, de Jong PTVM. The Prevalence of Primary Open-angle Glaucoma in a Populationbased Study in The Netherlands. Ophthalmology. 1994;101:1851-5. https:// doi.org/10.1016/s0161-6420(94)31090-6.

12. Chan MPY, Broadway DC, Khawaja AP, Yip JLY, Garway-Heath DF, Burr JM, et al. Glaucoma and intraocular pressure in EPIC-Norfolk Eye Study: cross sectional study. BMJ. 2017:j3889. https://doi.org/10.1136/bmj.j3889. 
13. Janssen SF, Gorgels TGMF, Ramdas WD, Klaver CCW, van Duijn CM, Jansonius NM, et al. The vast complexity of primary open angle glaucoma: disease genes, risks, molecular mechanisms and pathobiology. Prog Retin Eye Res. 2013;37:31-67.

14. Kapetanakis W, Chan MPY, Foster PJ, Cook DG, Owen CG, Rudnicka AR. Global variations and time trends in the prevalence of primary open angle glaucoma (POAG): a systematic review and meta-analysis. Br J Ophthalmol. 2016;100(1):86-93.

15. Maxwell WJ, Glover GJ. Principles and Practice of Screening for Disease. Ann Intern Med. 1968;69:1085. https://doi.org/10.7326/0003-4819-69-5-1085_2.

16. Burr JM, Mowatt G, Hernández R, Siddiqui MAR, Cook J, Lourenco T, et al. The clinical effectiveness and cost-effectiveness of screening for open angle glaucoma: a systematic review and economic evaluation. Health Technol Assess. 2007;11(41):iii-iv, ix-X, 1-190

17. Mowatt G, Burr JM, Cook JA, Siddiqui MAR, Ramsay C, Fraser C, et al. Screening tests for detecting open-angle glaucoma: systematic review and meta-analysis. Invest Ophthalmol Vis Sci. 2008;49(12):5373-85.

18. Armaly MF. Biostatistical Analysis of the Collaborative Glaucoma Study. Arch Ophthalmol. 1980;98:2163. https://doi.org/10.1001/archopht.1980. 01020041015002.

19. Wolfs RC, Klaver CC, Ramrattan RS, van Duijn CM, Hofman A, de Jong PT. Genetic risk of primary open-angle glaucoma. Population-based familial aggregation study. Arch Ophthalmol. 1998;116(12):1640-5.

20. Marcus MW, de Vries MM, Junoy Montolio FG, Jansonius NM. Myopia as a risk factor for open-angle glaucoma: a systematic review and meta-analysis. Ophthalmology. 2011;118(10):1989-94.e2.

21. Khawaja AP, Viswanathan AC. Are we ready for genetic testing for primary open-angle glaucoma? Eye. 2018;32(5):877-83.

22. Asefa NG, Neustaeter A, Jansonius NM, Snieder H. Heritability of glaucoma and glaucoma-related endophenotypes: Systematic review and metaanalysis. Surv Ophthalmol. 2019;64:835-51. https://doi.org/10.1016/j. survophthal.2019.06.002.

23. Sears NC, Boese EA, Miller MA, Fingert JH. Mendelian genes in primary open angle glaucoma. Exp Eye Res. 2019;186:107702

24. Iglesias Al, Springelkamp H, Ramdas WD, Klaver CCW, Willemsen R, van Duijn CM. Genes, pathways, and animal models in primary open-angle glaucoma. Eye. 2015;29(10):1285-98.

25. Springelkamp H, Iglesias Al, Mishra A, Höhn R, Wojciechowski R, Khawaja AP et al. New insights into the genetics of primary open-angle glaucoma based on meta-analyses of intraocular pressure and optic disc characteristics. Hum Mol Genet. 2017;26(2):438-53.

26. Craig JE, Han X, Qassim A, Hassall M, Cooke Bailey JN, Kinzy TG, et al. Multitrait analysis of glaucoma identifies new risk loci and enables polygenic prediction of disease susceptibility and progression. Nat Genet. 2020;52(2):160-6.

27. Choquet H, Paylakhi S, Kneeland SC, Thai KK, Hoffmann TJ, Yin J, et al. A multiethnic genome-wide association study of primary open-angle glaucoma identifies novel risk loci. Nat Commun. 2018;9(1):2278.

28. Genetic risk scores in complex eye disorders. In: Genetics and Genomics of Eye Disease: Academic Press; 2020. p. 259-75.

29. Klijs B, Scholtens S, Mandemakers JJ, Snieder H, Stolk RP, Smidt N. Representativeness of the LifeLines cohort study. PLoS One. 2015;10(9): e0137203.

30. Stolk RP, Rosmalen JGM, Postma DS, de Boer RA, Navis G, Slaets JPJ, et al. Universal risk factors for multifactorial diseases: LifeLines: a three-generation population-based study. Eur J Epidemiol. 2008;23(1):67-74.

31. Scholtens S, Smidt N, Swertz MA, Bakker SJL, Dotinga A, Vonk JM, et al. Cohort profile: LifeLines, a three-generation cohort study and biobank. Int J Epidemiol. 2015;44(4):1172-80.

32. Purcell S, Neale B, Todd-Brown K, Thomas L, Ferreira MAR, Bender D, et al. PLINK: a tool set for whole-genome association and population-based linkage analyses. Am J Hum Genet. 2007;81(3):559-75.

33. Browning BL, Browning SR. A unified approach to genotype imputation and haplotype-phase inference for large data sets of trios and unrelated individuals. Am J Hum Genet. 2009:84(2):210-23.

34. Consortium the International Hapmap, †The International HapMap Consortium. The International HapMap Project. Nature. 2003;426:789-96. https://doi.org/10.1038/nature02168.

35. Nolte IM, van der Most PJ, Alizadeh BZ, de Bakker PIW, Marike Boezen H Bruinenberg $M$, et al. Missing heritability: is the gap closing? An analysis of 32 complex traits in the Lifelines Cohort Study. Eur J Hum Genet. 2017;25: 877-85. https://doi.org/10.1038/ejhg.2017.50.
36. Fadista J, Manning AK, Florez JC, Groop L. The (in)famous GWAS P-value threshold revisited and updated for low-frequency variants. Eur J Hum Genet. 2016;24:1202-5. https://doi.org/10.1038/ejhg.2015.269.

37. Machiela MJ, Chanock SJ. LDlink: a web-based application for exploring population-specific haplotype structure and linking correlated alleles of possible functional variants. Bioinformatics. 2015;31(21):3555-7.

38. Ogbuehi KC, Almubrad TM. Evaluation of the Intraocular Pressure Measured with the Ocular Response Analyzer. Curr Eye Res. 2010;35:587-96. https:// doi.org/10.3109/02713681003698871.

39. Paudel N, Adhikari S, Thakur A, Shrestha B, Loughman J. Clinical accuracy of the Nidek ARK-1 autorefractor. Optom Vis Sci. 2019:96(6):407-13.

40. Maddess T, Goldberg I, Dobinson J, Wine S, Welsh AH, James AC. Testing for glaucoma with the spatial frequency doubling illusion. Vis Res. 1999:39: 4258-73. https://doi.org/10.1016/s0042-6989(99)00135-2.

41. Johnson CA, Samuels SJ. Screening for glaucomatous visual field loss with frequency-doubling perimetry. Invest Ophthalmol Vis Sci. 1997;38(2):413-25.

42. Stoutenbeek $\mathrm{R}$, Heeg GP, Jansonius NM. Frequency doubling perimetry screening mode compared to the full-threshold mode. Ophthalmic Physiol Opt. 2004;24(6):493-7.

43. de Vries MM, Stoutenbeek R, Müskens RPHM, Jansonius NM. Glaucoma screening during regular optician visits: the feasibility and specificity of screening in real life. Acta Ophthalmol. 2012;90(2):115-21.

44. Höhn R, Kottler U, Peto T, Blettner M, Münzel T, Blankenberg S, et al. The ophthalmic branch of the Gutenberg health study: study design, cohort profile and self-reported diseases. PLoS One. 2015;10(3):e0120476.

45. Wollstein G, Kagemann L, Bilonick RA, Ishikawa H, Folio LS, Gabriele ML, et al. Retinal nerve fibre layer and visual function loss in glaucoma: the tipping point. Br J Ophthalmol. 2012;96(1):47-52.

46. Wolfs RC, Borger PH, Ramrattan RS, Klaver CC, Hulsman CA, Hofman A, et al. Changing views on open-angle glaucoma: definitions and prevalences--the Rotterdam study. Invest Ophthalmol Vis Sci. 2000;41(11):3309-21.

47. Broadway DC, Nicolela MT, Drance SM. Optic disk appearances in primary open-angle glaucoma. Surv Ophthalmol. 1999;43(Suppl 1):S223-43.

48. Foster PJ, Buhrmann R, Quigley HA, Johnson GJ. The definition and classification of glaucoma in prevalence surveys. Br J Ophthalmol. 2002; 86(2):238-42.

49. Ramdas WD, Rizopoulos D, Wolfs RCW, Hofman A, de Jong PTVM, Vingerling $J R$, et al. Defining glaucomatous optic neuropathy from a continuous measure of optic nerve damage - the optimal cut-off point for risk-factor analysis in population-based epidemiology. Ophthalmic Epidemiol. 2011;18(5):211-6.

50. Karvonen E, Stoor K, Luodonpää M, Hägg P, Kuoppala J, Lintonen T, et al. Prevalence of glaucoma in the northern Finland birth cohort eye study. Acta Ophthalmol. 2019:97(2):200-7.

51. Tielsch JM, Katz J, Sommer A, Quigley HA, Javitt JC. Family history and risk of primary open angle glaucoma. The Baltimore Eye Survey. Arch Ophthalmol. 1994;112(1):69-73

52. Igo RP, Cooke Bailey JN. Genetic risk scores in complex eye disorders. Genet Genomics Eye Dis. 2020:259-75. https://doi.org/10.1016/b978-0-12-816222-4. 00016-2.

\section{Publisher's Note}

Springer Nature remains neutral with regard to jurisdictional claims in published maps and institutional affiliations.

Ready to submit your research? Choose BMC and benefit from

- fast, convenient online submission

- thorough peer review by experienced researchers in your field

- rapid publication on acceptance

- support for research data, including large and complex data types

- gold Open Access which fosters wider collaboration and increased citations

- maximum visibility for your research: over $100 \mathrm{M}$ website views per year

At $\mathrm{BMC}$, research is always in progress.

Learn more biomedcentral.com/submissions 